\title{
TOLERANCE INDUCED WITHOUT IMMUNOSUPPRESSION IN A T-LYMPHOCYTE SUICIDE-GENE THERAPY CARDIAC ALLOGRAFT MODEL IN MICE
}

\author{
E. Braunberger \\ N. Raynal-Raschilas \\ V. Thomas-Vaslin \\ P. Bruneval \\ J. N. Fabiani \\ A. Carpentier \\ D. Glotz \\ D. Klatzmann
}

Background: Life-long immunosuppression is a major cause of mortality and morbidity in transplant recipients. Gene therapy could provide new ways to obtain tolerance and avoid indefinite immunosuppression. EpTK mice are derived from the FVB/N strain $(\mathrm{H} 2 q)$ and express the thymidine kinase gene of herpesvirus in all mature $T$ cells. Thus any mature dividing $\mathbf{T}$ cell can be killed in the presence of ganciclovir. We investigated the survival of alloincompatible C57B1/6 (H2b) hearts heterotopically transplanted into EpTK mice given only ganciclovir from day 0 to day 7 or 14. Methods: Abdominal cardiac transplantations were performed in 22 control mice (untreated FVB [ $n=15]$, ganciclovir-treated FVB $[n=5]$, and untreated EpTK mice $[n=2])$ and in 28 EpTK mice given ganciclovir from day 0 to day $7(n=15)$ or day $14(n=13)$. Rejection was defined as complete cessation of cardiac beat. Histologic examination of the grafts was performed at rejection, at day 7 , or at day 100. Lymphocyte proliferation assays (concanavalin A stimulation or mixed lymphocyte reaction) were performed at day 7 and at day 100 . Results: All control animals rejected transplants in 7 days (range, 5-9 days), whereas indefinite survival (>100 days) was observed in $89 \%$ of the ganciclovir-treated EpTK group, irrespective of the duration of ganciclovir treatment. Graft histology showed extensive cellular infiltrates with myocyte necrosis and arteritis in the control animals but only a mild infiltrate without necrosis or arteritis in the ganciclovir-treated EpTK group. The proliferative responses of the tolerant mice at day 100 were identical to those of naive mice, including a preserved proliferation against the donor's lymphocytes in mixed lymphocyte reaction. Conclusion: Functional transplantation tolerance of a fully incompatible heart can be achieved without immunosuppressive drugs in this model of suicide gene therapy. (J Thorac Cardiovasc Surg 2000;119:46-52)
C linical results of allotransplantation have improved dramatically in recent years, with 1-year graft survival of $70 \%$ to $80 \%$. $^{1}$ The need for lifelong immunosuppression, however, remains a major limitation and is

From the Laboratoire d'etude des greffes et protheses cardiaques, Hopital Broussais, Paris, France.

Read at the Seventy-ninth Annual Meeting of The American Association for Thoracic Surgery, New Orleans, La, April 18-21, 1999.

Received for publication April 22, 1999; revisions requested June 21, 1999; revisions received Sept 8, 1999; accepted for publication Sept 16, 1999.

Address for reprints: Professor Denis Glotz, INSERM U430, Hopital Broussais, 96 rue Didot, 75014, Paris, France, and Dr Eric Braunberger, Laboratoire d'etude des greffes et protheses cardiaques, Hopital Broussais, 96 rue Didot, 75014, Paris, France.

Copyright (C) 2000 by Mosby, Inc.

$0022-5223 / 2000 \$ 12.00+0 \quad \mathbf{1 2 / 6 / 1 0 3 0 1 4}$ associated with certain risks to the patient. In particular, organ-transplant recipients have a higher risk of opportunistic infections ${ }^{2}$ and malignancies, such as lymphoma. ${ }^{3}$ Although improvements in immunosuppressive therapy have significantly decreased the incidence and morbidity of acute rejection episodes, chronic rejection resulting in graft failure continues to limit the long-term efficacy of allotransplantation., ${ }^{4,5}$ The induction of donor-specific immune tolerance would, in theory, eliminate the need for immunosuppression and its associated problems. It has long been a major goal of transplant immunology research.

Although allograft rejection is a complex phenomena involving multiple cell types and soluble factors, T lymphocytes play a central role in the process. ${ }^{6,7}$ The alloreactive $\mathrm{T}$ lymphocyte is therefore a natural target for transplantation immunotherapy. ${ }^{8}$ Conventional treat- 
Volume 119, Number 1

Table I. Results of heart survival

\begin{tabular}{llllr}
\hline Group & Recipients & Treatment & Survival $(d)$ & MST $(d)$ \\
\hline A & FVB/N (10) & None & $6(\times 3), 7(\times 6), 9$ & 7 \\
B1 & Littermates (5) & Ganciclovir-7 days & $6,7(\times 4)$ & 7 \\
B2 & Littermates (5) & None & $5,6,7(\times 3)$ & 7 \\
C1 & EpTK (4) & Ganciclovir-7 days & $26,>100(\times 3)$ & $>100$ \\
C1 & EpTK $(10)^{*}$ & Ganciclovir-14 days & $15,37,>100(\times 8)$ & $>100$ \\
C2 & EpTK (2) & None & $7(\times 2)$ & 7 \\
\hline
\end{tabular}

All donors were from C57B1/6 origin.

$M S T$, Mean survival time.

${ }^{*}$ The 4 mice put to death at day 7 and the 10 dead are not reported.

ments use mostly antibodies directed at various cell antigens or drugs affecting the activation and multiplication of B and T cells.

Another strategy would be to use the possibilities offered by gene therapy and, more specifically, a suicide-gene system. The TK suicide-gene system uses the gene for herpes simplex virus 1 thymidine kinase. Unlike TK from eukaryotic cells, TK of the virus can phosphorylate nucleoside analogues such as ganciclovir. Monophosphated ganciclovir is then phosphorylated by the eukaryotic cells to triphosphate ganciclovir, which is incorporated into dividing cells and stops DNA synthesis, thus leading to cell death. ${ }^{9}$

Destruction of cells is thus restricted to dividing cells and conditioned by the presence of ganciclovir. To test the efficacy of this system in the therapy of T-cell mediated diseases, we generated a strain of mice, the transgenic mouse line EpTK, in which a TK transgene is only expressed in T lymphocytes. ${ }^{10,11}$ The regulating sequences come from the human CD4 gene, the promoter of which is remarkably similar in sequence to murine promoters, and from the murine CD4 gene enhancer. $^{12}$

This system destroys $\mathrm{T}$ lymphocytes in a selective (replicating cells) and conditional (in the presence of ganciclovir) way.

\section{Methods}

Animals. All mice were aged 6 to 15 weeks and weighed 15 to $30 \mathrm{~g}$. Donor mice were male C57B1/6 animals of the H$2^{\mathrm{b}}$ haplotype (CERJ laboratory, Le Genest-Saint-Isle, France). Recipient mice were either FVB mice of the H-2 haplotype (IFFA Credo laboratory, Domaine des Oncins, L'Arbesle, France) or transgenic EpTK mice (derived from the FVB strain and raised in the facility of the PitieSalpetriere Hospital, Paris, France). Third-party mice were $\mathrm{BALB} / \mathrm{c}$ of the $\mathrm{H}-2^{\mathrm{d}}$ haplotype (CERJ laboratory). All animals received humane care in compliance with the guide for the care and use of laboratory animals.

Murine heterotopic heart transplantation. Intra-abdom- inal heterotopic cardiac transplantation was performed by using techniques similar to those described by Corry and colleagues. ${ }^{13}$ The donor mouse was anesthetized with 100 $\mathrm{mg} / \mathrm{kg}$ intraperitoneal pentobarbital (Sanofi). The mouse was prepped with $90 \%$ alcohol, and a full midline laparotomy was performed under full sterile conditions. After administering $250 \mathrm{IU}$ of heparin into the inferior vena cava, the incision was extended into a median sternotomy. Under $10 \times$ magnification, the inferior vena cava was ligated with a $9 / 0$ monofilament (Premio, Peters) suture and divided distally. Under $20 \times$ magnification, the aorta and pulmonary artery were separated and divided as far distally as possible. The superior vena cava and pulmonary veins were then ligated en masse with a 6-0 silk suture. The heart was removed and stored in cold Ringer's lactate solution $\left(0^{\circ} \mathrm{C}-4^{\circ} \mathrm{C}\right)$ with antibiotics $(250,000 \mathrm{IU}$ penicillin plus $100 \mathrm{mg}$ gentamicin in $500 \mathrm{~mL}$ of solution).

The recipient mouse was anesthetized with $50 \mathrm{mg} / \mathrm{kg}$ intraperitoneal pentobarbital and $2.5 \mu \mathrm{g}$ of intraperitoneal atropine sulfate. Cefazolin, $25 \mathrm{mg} / \mathrm{kg}$ administered intramuscularly, was administered for antibiotic prophylaxis. Under identical sterile conditions, a full midline laparotomy was performed, and the intestines were retracted with an antibiotic-soaked sponge. Under $20 \times$ magnification, the infrarenal aorta and inferior vena cava were dissected over a length of 5 $\mathrm{mm}$. Lumbar branches were ligated by using 9-0 polyvinylidene difluoride monofilament (Premio, Peters). The aorta and vena cava were then encircled proximally and distally by using 6-0 silk ligatures and occluded by using a "meunier" knot. Under $40 \times$ magnification, a longitudinal venotomy and aortotomy were performed with a 26-gauge needle.

After the donor heart was removed from storage, the donor aorta and pulmonary artery were anastomosed to the recipient aorta and vena cava, respectively, by using a continuous running 10-0 Ethicon suture (Ethicon, Inc, Somerville, NJ). The anastomoses took about 20 minutes to complete, and the ischemic heart was protected by dripping cold lactated Ringer's solution on it at frequent intervals. The distal occluding ties were removed from the recipient's aorta and vena cava, followed by the proximal ties. After a brief period of ventricular fibrillation, the heart usually reverted spontaneously to normal sinus rhythm. After carefully replacing the 

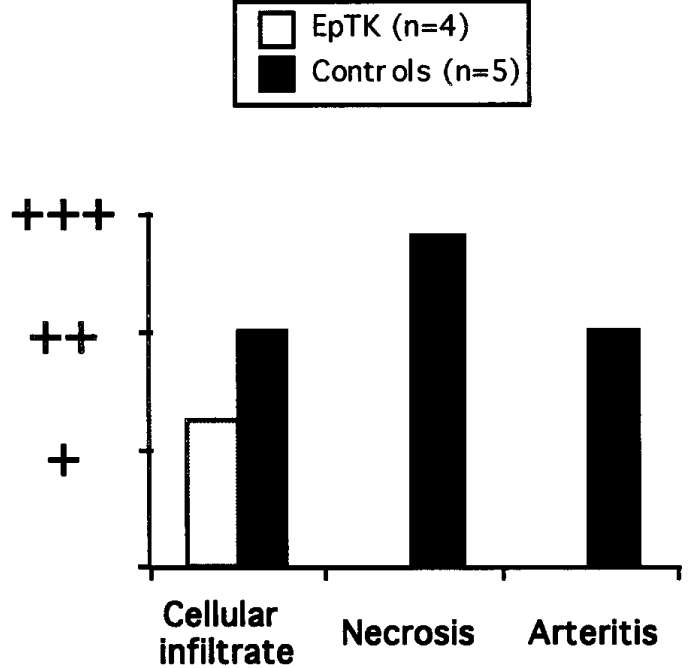

Fig 1. Histologic results. Comparison between ganciclovirtreated EpTK mice and control mice. Results are given in means of score for each data.

intestines in the abdomen and instilling $0.5 \mathrm{~mL}$ of lactated Ringer's solution with antibiotics, the abdominal wall was closed with 2 running sutures. Graft function was monitored daily by 2 separate investigators with direct palpation, and rejection was suspected when a sharp decline in the intensity of the cardiac impulse was noted. Rejection never led to death of the recipient animal.

Ganciclovir administration. Ganciclovir (Cymevan, Roche) was administered by continuous subcutaneous infusion initiated immediately postoperatively, with a miniosmotic pump Alzet (Alza Corp, Palo Alto, Calif) at a rate of $1 \mu \mathrm{L} / \mathrm{h}$ ( $50 \mathrm{mg} / \mathrm{kg}$ per day). The treatment was continued for 7 days (model 2001) or 14 days (model 2002).

Histology. All hearts were excised from the recipients and subjected to microscopic examination. Five-micrometer paraffin sections of ventricular tissue were stained with hematoxylin-eosin-safran stain and examined histologically. The samples were graded for cellular infiltrate, necrosis, and arteritis by 2 separate, blinded investigators on a scale of 0 to $3+$.

Mixed lymphocyte culture and concanavalin A stimulation. The assay medium was RPMI-1640 medium (Bio Whittaker, Fontenay-sous-bois, France) supplemented with 2 $\mathrm{mmol} / \mathrm{L} \mathrm{L}$-glutamine, $1 \%$ penicillin-streptomycin-neomycin, $50 \mu \mathrm{mol} / \mathrm{L} \quad \beta_{2}$-mercaptoethanol (Life Technologies-Gibco BRL, Cergy-Pontoise, France), and $10 \%$ fetal calf serum (Dominique Dutscher SA, Brumath, France).

Cells used in these experiments were splenocytes from the different mice strains. Splenocytes were gently passed through a $70-\mu \mathrm{m}$ cell strainer (Falcon; Becton Dickinson, Le Pont de Claix, France), and erythrocytes were lysed by treatment with an ammonium chloride and potassium solution.

A total of 100,000 responder cells were stimulated in triplicate in round-bottomed, 96-well microplates in $0.2 \mathrm{~mL}$ of assay medium with $3 \mu \mathrm{g} / \mathrm{mL}$ concanavalin A (Sigma-Aldrich, Saint-Quentin-Fallavier, France).

For the mixed lymphocyte culture, responder cells at the same concentration were stimulated by the addition of 200,000 splenocytes treated with mitomycin C (SigmaAldrich, Saint-Quentin-Fallavier, France).

Cells were cultured in a $5 \% \mathrm{CO}_{2}$ incubator at $37^{\circ} \mathrm{C}$.

Proliferation was assessed by incorporation of $1 \mu \mathrm{Ci}$ of ${ }^{3} \mathrm{H}$-thymidine (ICN Pharmaceuticals, Orsay, France) per well after 2 days of culture for the mitogen stimulation or after 4 days for the mixed lymphocyte culture. Cells were pulsed for 18 to 24 hours before harvesting. Radioactivity was counted by using the Tomtec 96-well harvester and the 1450 microbeta Plus-liquid scintillation counter, and the results were recorded as mean counts per minute.

Statistical analysis. Data are presented as means \pm SEM. Statistical significance was assessed by using analysis of variance and the Student unpaired $t$ test or Kaplan-Meier survival curves.

\section{Results}

Graft survival. We performed 50 abdominal heterotopic cardiac transplantations with C57B1/6 mice as donors (Table I).

Group A, B1, and B2 recipients were nontransgenic FVB mice. Group $\mathrm{C}$ recipients were transgenic mice treated by ganciclovir $(\mathrm{C} 1)$ or untreated $(\mathrm{C} 2)$.

Ten mice died in group $\mathrm{C} 1$ (on day 2, 6, 11, 18, 27, $32,35,50,52$, and 62 ) with no evident cause of death at systematic autopsy, including no evidence of sepsis or neoplasia, and with a strongly beating heart the day before. Four mice were killed at day 7 for histology.

Treatment with ganciclovir 7 or 14 days after transplantation in EpTK mice significantly prolonged allograft survival to greater than 100 days in 11 of 14 animals. In contrast, control animals consistently rejected their grafts between days 6 and $9(P<.0001)$.

Three rejections were observed in the $\mathrm{C} 1$ group (EpTK mice treated with ganciclovir). No difference was found between the groups treated for 7 or 14 days with ganciclovir $(P=.39$; Table I).

Macroscopic appearance. Rejecting cardiac allografts had a characteristic macroscopic appearance. They were dilated, mottled with dark coloration, and exhibited no visible beating. In contrast, nonrejecting grafts were small with normal coloration and exhibited strong and visible contractions.

Histology. The histologic appearance of allografts from the ganciclovir-treated EpTK group was compared with that of allografts from the control groups. Histologic evaluation was done between 6 and 8 days after transplantation (4 EpTK mice were killed at day 7). Histology of the hearts from acutely rejecting 


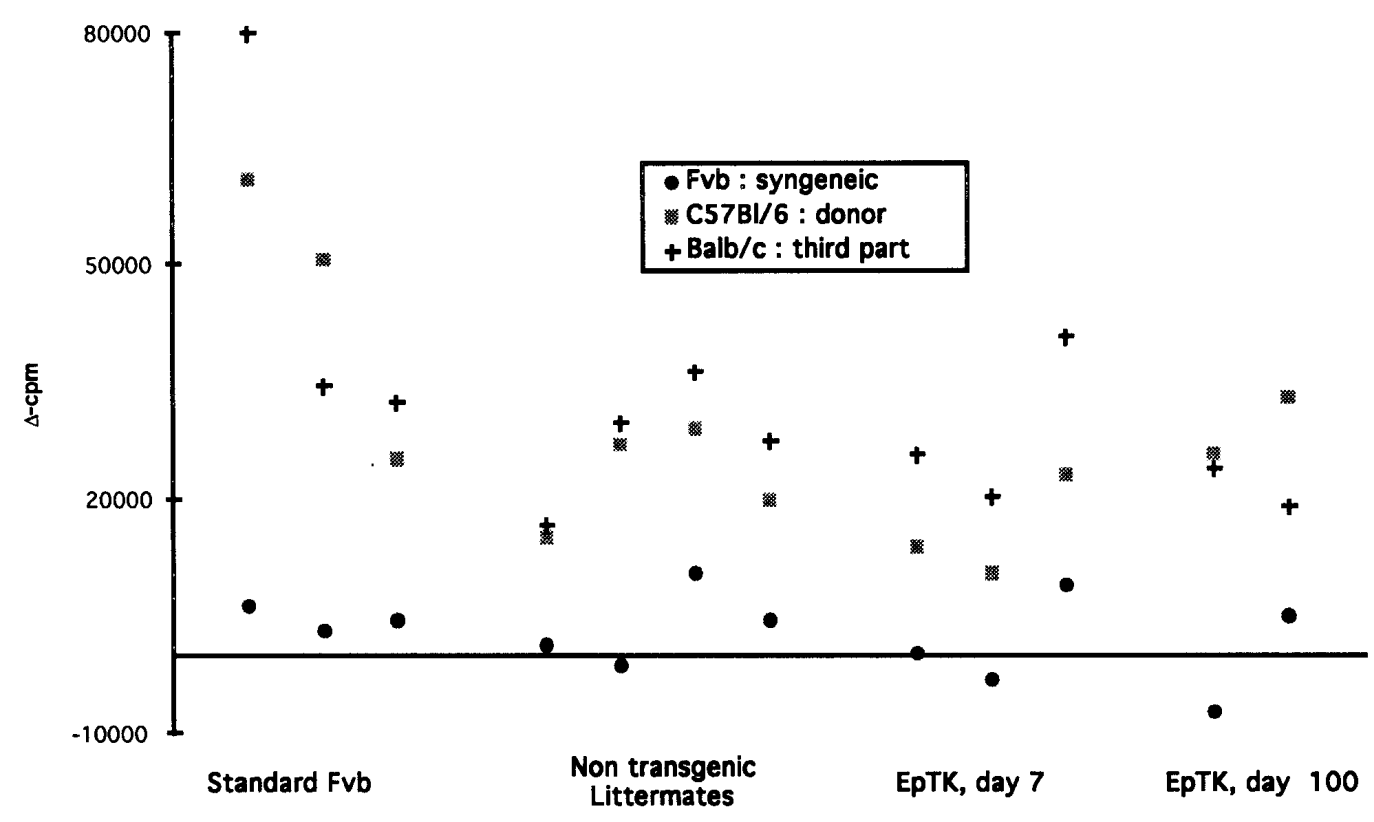

Fig 2. Mixed lymphocyte reaction of splenocytes after stimulation with donor and third-party antigens.

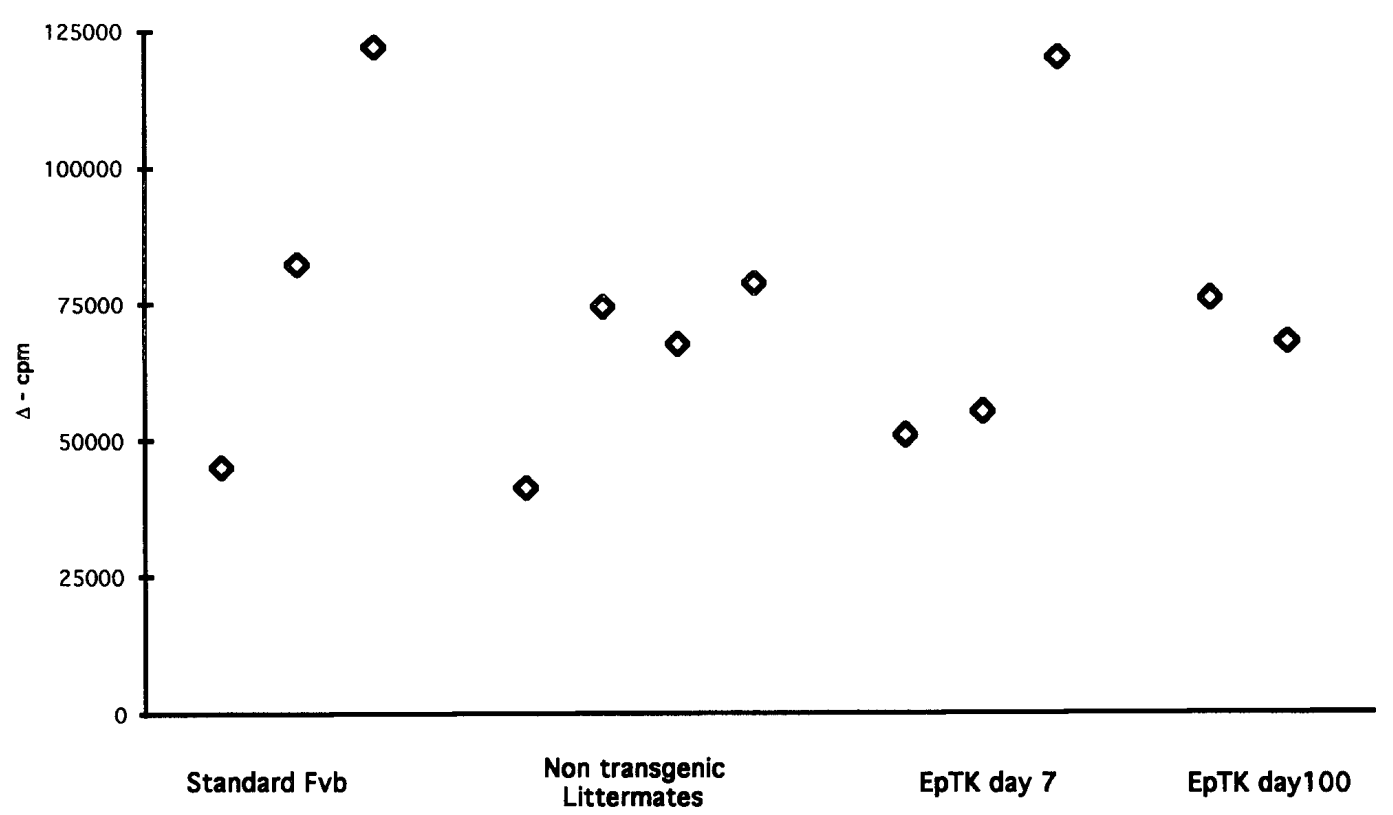

Fig 3. In vitro responsiveness of splenocytes stimulated by a nonspecific stimulant (mitogen) concanavalin A.

animals (control animals) demonstrated a marked cellular graft infiltrate with destruction of myocyte architecture.

Heart transplants from ganciclovir-treated EpTK mice also had a marked cellular infiltrate but with preserved myocyte architecture. The average rejection grade in the ganciclovir-treated EpTK group was between $1 \mathrm{~A}$ and 1B; the control group, on the other hand, demonstrated significant necrosis and arteritis with grade 4 rejection in all animals.

Fig 1 shows the histologic aspect of allograft at day 7 after transplantation for each group. 
Of the 7 mice who had standard histology between day 27 and day 101, 5 showed no necrosis at all, and 2 showed very mild necrosis. Six showed no arteritis, whereas 1 mouse killed at day 100 had some arteritis. The cellular infiltrate was present in most recipients, but it does not appear to destroy cardiac architecture.

Mixed lymphocyte culture and concanavalin A stimulation. The mixed lymphocyte reaction of cells from treated mice and control mice (naive FVB/N mice and treated transplanted nontransgenic litter mates) after stimulation with donor and third-party antigens were evaluated. Results are shown in Fig 2.

The proliferative response in ganciclovir-treated EpTK mice killed at day 7 was partially inhibited when stimulated by donor cells in comparison with third-party cells, but by day 100 after transplantation, these mice responded normally to donor and thirdparty antigens.

We also studied in vitro responsiveness of splenocytes from the same mice groups stimulated by concanavalin A (Fig 3).

Splenocytes from ganciclovir-treated EpTK mice killed at days 7 or 100 exhibited a strong proliferative response to mitogen.

Lymphocyte count. Blood cell count realized at the seventh day after transplantation on 6 ganciclovir-treated EpTK mice revealed a normal lymphocyte count (average of $3300 / \mathrm{mm}^{3}[ \pm 1230]$ compared with levels in control mice of $3480 / \mathrm{mm}^{3}[ \pm 520]$ ).

\section{Discussion}

The suicide-gene system used in this study attempts to induce donor-specific tolerance by targeting replicating $\mathrm{T}$ lymphocytes for self-destruction during the first days after transplantation. By genetically modifying the recipient's T lymphocytes, we have acquired the ability to selectively destroy replicating $\mathrm{T}$ lymphocytes during ganciclovir administration. The assumption is that during this early postoperative period, most replicating $\mathrm{T}$ lymphocytes will be alloreactive and, conversely, that most alloreactive $\mathrm{T}$ lymphocytes will be replicating (and therefore suicidal). We hypothesized that we could successfully eliminate the pool of alloreactive $\mathrm{T}$ lymphocytes during this period and consequently induce long-term specific tolerance to the allograft. Indeed, Cohen and colleagues ${ }^{11}$ used that same strain of mice in a bone-marrow transplant model. The investigators subjected nontransgenic mice to lethal irradiation and reconstituted the mice with allogeneic $\mathrm{T}$ cell-deplated bone marrow and allogeneic $\mathrm{T}$ cells from transgenic mice. Subsequent treatment with a 7-day course of ganciclovir prevented graft-versus-host dis- ease (GVHD). The pool of alloreactive mature lymphocytes that were reactive to the host (ie, those causing GVHD) was apparently successfully destroyed by the ganciclovir treatment, rendering the grafted allogeneic hematopoietic cells incapable of causing GVHD. The specific nature of this protection was confirmed by the fact that donor $\mathrm{T}$ lymphocytes were identified in the recipient that reacted normally to thirdparty cells. These lymphocytes, of course, were specifically tolerant of the recipient.

Our results, using a vascularized graft model, demonstrate a dramatic increase in cardiac allograft survival in the ganciclovir-treated transgenic mice. Because ganciclovir-treated nontransgenic mice (group B1), as well as untreated transgenic mice (group C2), rejected their grafts, as did the untreated controls, we can rule out an immunosuppressive effect of the ganciclovir itself or an intrinsic immunodeficiency of the transgenic mice.

There was little histologic evidence of myocardial injury in ganciclovir-treated transgenic mice when compared with 4 different control groups. At approximately 1 week, the histologic pattern is quite clear, with significant cellular infiltration, necrosis, and arteritis in all control groups. In contrast, the ganciclovir-treated transgenic mice showed only mild cellular infiltration without evidence of necrosis or arteritis. All control mice rejected their allografts within 9 days. Although 3 ganciclovir-treated transgenic mice rejected their grafts between 15 and 37 days (rejection related to inadequate delivery of ganciclovir), the remaining 11 continued to function until they were killed at more than 100 days. Although a mild cellular infiltrate persisted in these long-term survivors, necrosis and arteritis remained rare and, when present, quite mild. The significance of this persistent mild cellular infiltrate, without evidence of myocardial injury or graft dysfunction, is not clear from our study. However, it is a common finding in other models of allograft tolerance ${ }^{14,15}$ and may, in fact, consist of suppressive T lymphocytes that protect the allograft from rejection. ${ }^{16,17}$

Tolerance in this model is achieved without any longlasting immunodepletion, as shown by a normal lymphocyte count and normal proliferative responses in the tolerant mice. Both 7 and 14 days of ganciclovir treatment seemed to confer equal graft protection in the transgenic mice, suggesting that early events affecting the alloreactive lymphocytes have a long-lasting effect. Because tolerant mice have, at day 100, a normal proliferative response against the donor cells, a simple, time-limited, clonal deletion of alloreactive T lymphocytes cannot by itself explain the long-lasting tolerance 
seen. Future work identifying the immune mechanisms at play is clearly warranted.

This system could be used in patients through ex vivo transfection of bone marrow cells and subsequent autologous bone marrow transplantation at the time of solid organ transplantation.

In summary, we have induced a long-lasting functional tolerance in a murine model of vascularized cardiac allotransplantation without the use of any immunosuppressive agent. Although many hurdles will have to be overcome before this system could be applied to clinical transplantation, these promising early results appear to validate the principles on which the suicide-gene system is based.

\section{REFERENCES}

1. Calne R. Impact of immunosuppressants on host tissues and tolerance. Transplant Proc 1994;26:3065-70.

2. Patel R, Paya CV. Infections in solid-organ transplant recipients. Clin Microbiol Rev 1997;10:86-124.

3. Mihalov ML, Gattuso P, Abraham K, Holmes EW, Reddy V. Incidence of post-transplant malignancy among 674 solid-organtransplant recipients at a single center. Clin Transplant 1996; 10:248-55.

4. Shaikewitz T. Chronic renal transplant rejection. Am J Kidney Dis 1994;23:884-93.

5. Nagano H, Tilney NL. Chronic allograft failure: the clinical problem. Am J Med Sci 1997;313:305-9.

6. Fabre JW. Is tolerance a prospective for clinical research? Transpl Int 1992;5:S571-7.

7. Hall BM. Cells mediating allograft rejection. Transplantation 1991;51:1141-51.

8. Pattison JM. New insights into mechanisms of allograft rejection. Am J Med Sci 1997;313:257-63.

9. Roizman B. The function of herpes simplex virus genes: a primer for genetic engineering of novell vectors. Proc Natl Acad Sci USA 1996;93:11307-12.

10. Salmon P, Boyer O, Lores P., Jami J, Klatzmann D. Characterization of imtronless CD4 minigene expressed in mature CD4 and CD8 $\mathrm{T}$ cells, but not expressed in immature thymocytes. J Immunol 1996;156:1873-9.

11. Cohen JL, Boyer O, Salomon B, Onclercq R, Charlotte F, Bruel $\mathrm{S}$, et al. Prevention of graft-versus-host disease in mice using a suicide gene expressed in T lymphocytes. Blood 1997;89:463645.

12. Salmon P, Giovane A, Wasylyk B, Klatzmann D. Characterization of the human CD4 gene promoter: transcription from the CD4 gene core promoter is tissue-specific and is activated by Ets proteins. Proc Natl Acad Sci USA 1993;90:7739-45.

13. Corry RJ, Winn HJ, Russell PS. Primarily vascularized allografts of hearts in mice. Transplantation 1973;16:343-50.

14. Krieger NR, Most D, Bromberg JS, Holm B, Huie P, Sibley RK, et al. Coexistence of anti-TH1 and TH2-type cytokine profiles in anti-CD2 monoclonal antibody-induced tolerance. Transplantation 1996;62:1285-92.

15. Bugeon L, Cuturi MC, Hallet MM, Paineau J, Chabannes D, Soulillou JP. Peripheral tolerance of an allograft in adult ratscharacterization by low interleukin-2 and interferon-g mRNA levels and by strong accumulation of major histocompatibility complex transcripts in the graft. Transplantation 1992;54:219-25.

16. Takeuchi T, Lowry RP, Konieczny B. Heart allografts in murine systems. Transplantation 1992;53:1281-94.

17. Wu CJ, Lovett M, Wong-Lee J, Moeller F, Kitamura M, Goraalski TJ, et al. Cytokine gene expression in rejecting cardiac allografts. Transplantation 1992;54:326-32.

\section{Discussion}

Dr Verdi J. DiSesa (Chicago, Ill). You gave the ganciclovir for 7 days, is that what I saw on your slide?

Dr Braunberger. We gave ganciclovir for 7 days in 15 mice and for 14 days in 13 mice, with exactly the same survival rate.

Dr DiSesa. When did you start it relative to the transplant?

Dr Braunberger. We start immediately postoperatively, just before the mouse wakes up.

Dr DiSesa. Would you not hypothesize, though, that any stimulus that would lead to T-cell proliferation would be inhibited by this therapy?

Dr Braunberger. That is correct.

Dr DiSesa. So if the mouse was exposed to some infectious agent that ordinarily would cause his T-cell response to mount, that would be blunted as well.

Dr Braunberger. It appears that a treatment of 7 days is enough to induce tolerance, and we hope that in this 7-day period, the T-cell proliferation will be essentially alloreactive $\mathrm{T}$ cells. If infectious $\mathrm{T}$ cells are destroyed, we hope that it will be a limited phenomenon.

Dr DiSesa. So you think that that period of vulnerability would be confined to the period during which you are giving the ganciclovir?

Dr Braunberger. Yes, that is the fundamental principle of this model. If there is no ganciclovir, proliferative $\mathrm{T}$ cells are not destroyed.

Dr DiSesa. The problem in thinking how to apply this clinically is that making human transgenic recipients is probably going to be somewhat difficult.

Dr Braunberger. It may be possible to do bone marrow autotransplantation with a step of ex vivo transfection. This would be followed by organ transplantation, a short course of ganciclovir, and any other immunosuppressive treatment. Of course, we will need more data, and a primate experimental model will give valuable information before trying the treatment on human beings. This new strategy could be particularly interesting in transplantation of organs with a very high rate of rejection, like intestines or lungs.

Dr James L. Cox (Washington, DC). What happens after a hundred days?

Dr Braunberger. We killed most mice in this study, but we allowed a few to live more than 200 days with a beating heart. Because this strain of mice usually dies after 1 year, we were obliged to sacrifice them after that period.

Dr Cox. Do you ever re-administer ganciclovir?

Dr Braunberger. Never. We use a unique 7-day treatment.

Dr Cox. Would there be any advantage to re-administering it at 200 days or so? 
Dr Braunberger. Probably. Our goal was to demonstrate that it was possible to achieve tolerance only with a very short treatment. Of course, in human beings, it might be possible to treat rejection episodes with repeated short courses of ganciclovir.

Dr Paul Kurlansky (Miami Beach, Fla). I am just trying to understand what is going on in this magnificent work. Could you please explain what exactly is happening in terms of the DNA? Does the DNA message get incorporated permanently into the $\mathrm{T}$ cells and does it also get incorporated elsewhere in the genetic information, or is it incorporated in such a way that the T-cell mechanism uses this DNA, but it is not a permanent resident in the actual DNA structure of the cell?

Dr Braunberger. The gene is permanently incorporated into the genome. Because gene expression is controlled by CD4 promoter, it is expressed only in mature T cells.

\section{ONTHE MOVE?}

Don't miss a single issue of the journal! To ensure prompt service when you change your address, please photocopy and complete the form below.

Please send your change of address notification at least six weeks before your move to ensure continued service. We regret we cannot guarantee replacement of issues missed due to late notification.

\section{JOURNAL TITLE:}

Fill in the title of the journal here.

\section{OLD ADDRESS:}

Affix the address label from a recent issue of the journal here.

\section{NEW ADDRESS: \\ Clearly print your new address here.}

Name

Address

City/State/ZIP
COPY AND MAIL THIS FORM TO:

Periodical Subscription Services

Mosby, Inc.

11830 Westline Industrial Dr.

St. Louis, MO 63146-3318
OR FAX TO:

314-432-1158

N/M Mosby
OR PHONE:

1-800-453-4351

Outside the U.S., call

314-453-4351 